\title{
Metodologías de investigación de los semilleros E-InnovaCMM y TECSIS desde la virtualidad
}

Rubén Darío Cárdenas-Espinosa ${ }^{1}$

Servicio Nacional de Aprendizaje - SENA rdcardenas75@misena.edu.co

Luis Alfonso Devia-Caicedo

Servicio Nacional de Aprendizaje - SENA

ldeviac@sena.edu.co

Darwin Dubay Rodríguez-Pinto ${ }^{3}$

Servicio Nacional de Aprendizaje - SENA

drodriguezp@sena.edu.co

Claudia María Martínez-Zuluaga ${ }^{4}$

Servicio Nacional de Aprendizaje - SENA

cmartinezz@sena.edu.co

Fabio Andrés López-Salazar

Universidad de Caldas

fabio.lopez@ucaldas.edu.co

Jhon Jairo Ruiz-Salazar ${ }^{6}$

Servicio Nacional de Aprendizaje - SENA

jjruiz@misena.edu.co

DOI: https://doi.org/10.21158/2357514x.v8.n2.2020.2716

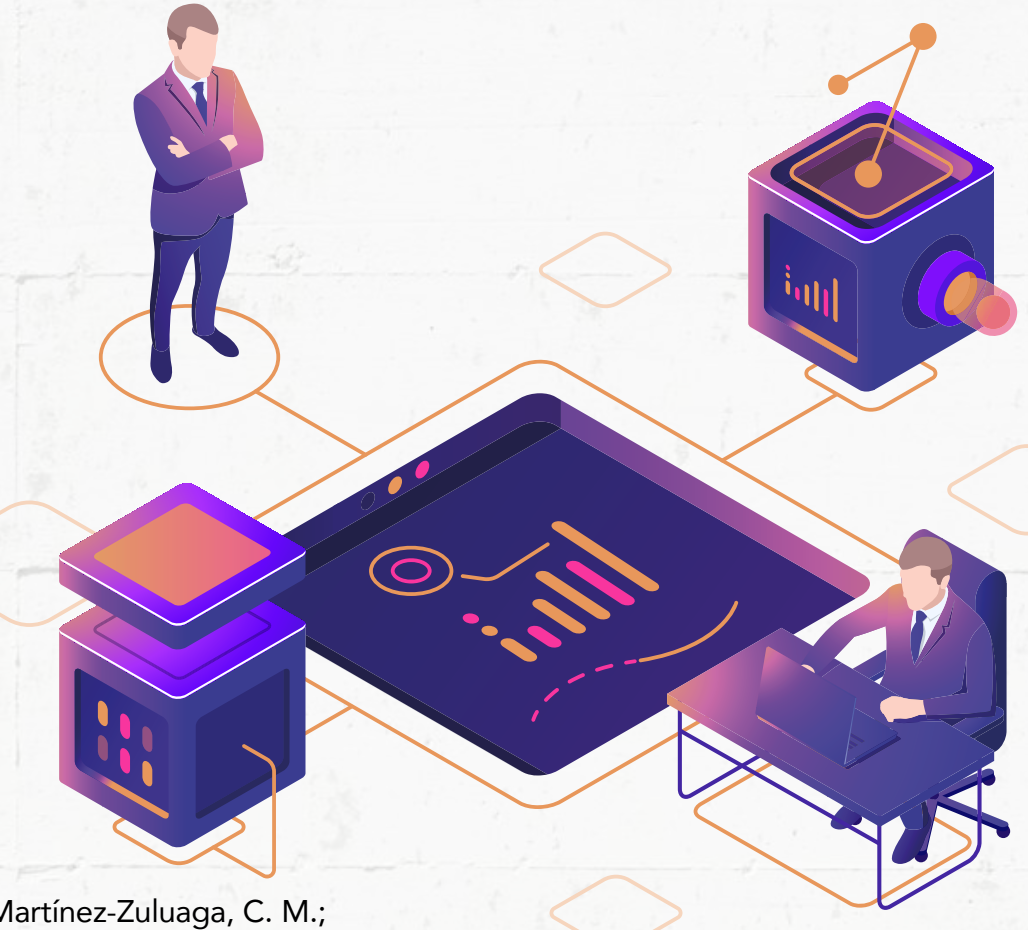

López-Salazar, F. A.; Ruiz-Salazar, J. J. (2020). Metodologías de investigación de los semilleros E-InnovaCMM y TECSIS

desde la virtualidad. Revista Virtu@Imente, 8(2), 29-45. DOI https://doi.org/10.21158/2357514x.v8.n2.2020.2716

Fecha de recepción: 02 de agosto de 2020

Fecha de aprobación: 09 de septiembre de 2020

Ingeniero Electrónico y Tecnólogo en Electrónica y Automatización Industrial - Universidad Autónoma de Manizales. Especialista en Gerencia de Finanzas - Universidad Autónoma de Manizales. Especialista Tecnológico en Interventoría de Proyectos de Telecomunicaciones - Servicio Nacional de Aprendizaje (SENA). Master of Sciences with major in Electrical Engineering - Atlantic International University. Doctor of Science with major in Electronic Engineering - Atlantic International University. Doctor of Philosophy with major in Technology Information - Atlantic International University. ORCID: http://orcid.org/0000-0002-2417-844X

2 Profesional en Ingeniería de Sistemas con énfasis en Administración - Universidad Ean. Diplomado en Herramientas de Gestión de Proyectos - Colegio de Estudios Superiores de Administración. Investigador Grupo de Investigación GICEMETORCID: https://orcid.org/0000-0002-8758-9203

${ }_{3}^{3}$ Ingeniero Electrónico - Universidad Distrital Francisco José de Caldas. Magister en Ingeniería Electrónica y de Computadores - Universidad de los Andes. ORCID: https://orcid.org/0000-0003-1172-1295 ${ }^{4}$ Ingeniera de Sistemas y Especialista en Gerencia de Proyectos - Universidad Ean. ORCID: https://orcid.org/0000-0003-2597-5739

${ }^{5}$ Ingeniero de Sistemas y Telecomunicaciones - Universidad Autónoma de Manizales. Especialista en Gerencia Empresarial - Universidad Autónoma de Manizales. Magister en Administración de Negocios - Universidad Autónoma de Manizales. ORCID: https://orcid.org/0000-0002-6943-8911

${ }^{6}$ Administrador de Empresas - Universidad Autónoma de Colombia. Especialista en Gerencia de Negocios Internacionales - Universidad Ean. Candidato a Magister Gestión estratégica de la Información y el Conocimiento - Universidad Pontificia Bolivariana. Líder Grupo de Investigación SENAGROQUIN del SENA Centro Agroindustrial Regional Quindío. ORCID: https://orcid.org/0000-0003-0260-4142 
El propósito de este artículo es socializar las metodologías de investigación aplicada e innovación que se han desarrollado de forma articulada desde la virtualidad, utilizando las Tecnologías de la Información y la Comunicación (TIC), en el Centro Metalmecánico Sena Distrito Capital, y en los programas especiales de la Facultad de Ingeniería de la Universidad de Caldas, promoviendo la investigación formativa en contexto según las necesidades identificadas en el entorno local de los aprendices. La metodología corresponde a una investigación mixta, de carácter analítico descriptivo, a partir de la cual se pretende cimentar proyectos posteriores que permitan profundizar en el conocimiento científico de unos campos específicos de conocimiento, aplicando el B-Learning a través del modelo PACIE (presencia, alcance, capacitación, interacción y e-learning). En el texto se describe el proceso que se implementó en los programas formativos y las trasformaciones que se dieron en los procesos y entornos investigativos de los estudiantes. Finalmente se evidencia cómo se lograron desarrollar recursos educativos digitales y transferencias tecnológicas desde el semillero E-InnovaCMM a los cinco semilleros de investigación de los programas especiales de la Universidad de Caldas, lo que demostró que el desarrollo de recursos educativos digitales puede facilitar el aprendizaje significativo y su uso en todos los niveles de formación —-tecnológico, técnico y complementario-.

Palabras clave: metodologías de investigación aplicada; investigación formativa; semilleros de investigación; B-Learning; Modelo PACIE; recursos educativos digitales; aprendizaje significativo; transferencia tecnológica. 


\section{Research methodologies used by E-InnovaCMM and TECSI seedbeds from the virtual setting}

\section{ABSTRACT}

This article socializes the applied innovative research methodologies developed from the virtual setting in an articulated way, using Information and Communication Technologies (ICT), from Centro Metalmecánico Sena Distrito Capital, and from the special programs of the Faculty of Engineering at Universidad de Caldas, promoting formative research in context according to the necessities identified in the local environment of the trainees. The methodology corresponds to a mixed research, of both analytical and descriptive character, intending to lay the foundations for subsequent projects that allow a deeper scientific knowledge of specific fields of knowledge, by applying the B-Learning through the Presence, Scope, Training, Interaction and E-learning model (PACIE by its abbreviation in Spanish). The text describes the process implemented in the training programs, and the transformations that took place in the processes and research environments of the students. Finally, it shows how digital educational resources and technological transfers were developed from the E-InnovaCMM seedbed in five research seedbeds of the special programs of Universidad de Caldas, which demonstrated that the development of digital educational resources can facilitate the significant learning and its use at all levels of training - technological, technical, and complementary-.

Keywords: applied research methodologies; formative research; research seedbeds; B-Learning; PACIE model; digital educational resources; significant learning; technology transfer. 


\title{
Metodologias de pesquisa dos grupos de pesquisa E-InnovaCMM e TECSIS a partir da virtualidade
}

\section{RESUMO}

\begin{abstract}
O objetivo deste artigo é socializar as metodologias de pesquisa aplicada e inovação que se têm desenvolvido de forma articulada a partir da virtualidade, recorrendo às Tecnologias de Informação e Comunicação (TIC), no Centro Metalúrgico Sena Distrito Capital, e nos programas especiais da Faculdade de Engenharia da Universidade de Caldas, promovendo a pesquisa formativa em contexto de acordo com as necessidades identificadas no meio local dos aprendizes. A metodologia corresponde a uma pesquisa mista, de natureza descritiva-analítica, a partir da qual se pretende cimentar projetos subsequentes que permitam aprofundar o conhecimento científico de áreas específicas do conhecimento, aplicando $B$-Learning através do modelo PACIE (presencial, avanço, capacitação, interação e e-learning). $\mathrm{O}$ texto descreve o processo que foi implementado nos programas de formação e as transformações ocorridas nos processos e ambientes de pesquisa dos alunos. Finalmente, fica evidente como se conseguiu desenvolver recursos educacionais digitais e transferências tecnológicas desde o grupo de pesquisa E-InnovaCMM para os cinco grupos de pesquisa dos programas especiais da Universidade de Caldas, o que demonstrou que o desenvolvimento de recursos educacionais digitais pode facilitar a aprendizagem significativa e sua utilização em todos os níveis de formação - tecnológico, técnico e complementar.
\end{abstract}

Palavras-chave: metodologias de pesquisa aplicada; pesquisa formativa; grupos de pesquisa; B-Learning; Modelo PACIE; recursos educacionais digitais; aprendizagem significativa; transferência de tecnologia. 


\section{Méthodologies de recherche en modalité virtuelle des pépinières d'entreprises E-InnovaCMM et TECSIS}

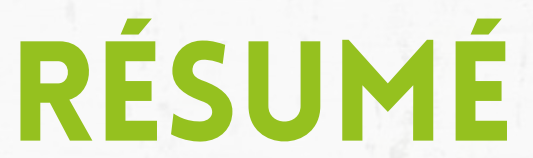

Cet article révèle les méthodologies de recherche appliquées à l'innovation et développées en modalité virtuelle à l'aide des technologies de l'information et de la communication (TIC) du SENA spécialisé en Mécanique Appliquée et au travers des programmes de la Faculté d'Ingénierie de l'Université de Caldas, en promouvant les investigations formatives en fonction des besoins identifiés dans l'environnement local des élèves et apprentis. II s'agit d'une méthodologie de recherche mixte, de type analytico-descriptive, ayant pour objectif de mettre en place de futurs projets permettant d'approfondir les connaissances scientifiques de domaines spécifiques du savoir via l'application du B-Learning au travers du modèle PACIE (présence, sensibilisation, formation, interaction et e-learning). L'article décrit le processus mis en œuvre dans les programmes de formation et les transformations observées dans les environnements de recherche des élèves. L'article décrira enfin la mise en place des ressources numériques et des transferts technologiques de la pépinière E-InnovaCMM vers les cing pépinières de recherche des programmes spéciaux de l'Université de Caldas, et mettra en évidence la façon dont ces ressources numériques peuvent faciliter un apprentissage significatif au travers d'une utilisation technologique, technique et complémentaire.

Mots-cléfs: méthodologies de recherche appliquée; recherche formative; pépinière de recherche; $\mathrm{B}$-Learning; Modèle PACIE; ressources pédagogiques et numériques; apprentissage significatif; transfert de technologie. 


\section{Introducción}

En este documento se presentará la investigación aplicada y el desarrollo tecnológico en el contexto de los programas especiales de la Universidad de Caldas, seguido de las tecnologías de la información para la investigación, la metodología y las respectivas conclusiones. El desarrollo de este proyecto ha contribuido a la inclusión educativa de los jóvenes desde las provincias profundas de los departamentos de Caldas, Cundinamarca y La Guajira, en donde hacen presencia las instituciones vinculadas.

Para este caso, se pudo aplicar la transferencia tecnológica de las estrategias didácticas activas y recursos educativos digitales desarrollados desde el semillero de investigación EInnovaCMM del Centro Metalmecánico Sena Distrito Capital, con el acompañamiento y la colaboración del grupo de investigación BIEMARC, de la Universidad de la Guajira, que conformó en febrero de 2017 el semillero de la Tecnología en Sistemas Informáticos (TECSIS); luego, el semillero de investigación RELEC en febrero de 2018, y en 2019 otros tres semilleros para los programas tecnológicos de la Facultad de Ingeniería de la Universidad de Caldas.

El desarrollo de recursos educativos digitales a partir de las capacitaciones recibidas por el Servicio Nacional de Aprendizaje (Sena), aplicando el modelo PACIE (Presencia, Alcance, Capacitación, Interacción y E-Learning), ha tenido como objetivo potenciar desde la virtualidad las estrategias y metodologías que fomenten la producción y divulgación científica desde los semilleros de investigación E-InnovaCMM, del Centro Metalmecánico, y TECSIS, de la Facultad de Ingeniería de Universidad de Caldas, que cubre los programas especiales Ingeniería Informática modalidad nocturna- y Tecnología en Sistemas Informáticos - modalidad a distancia-. Este trabajo colaborativo se ha realizado desde 2018 en cabeza del Centro Metalmecánico, con el apoyo de la Subdirección, el equipo directivo y el Sistema de Investigación, Desarrollo Tecnológico e Innovación (SENNOVA), y la Dirección del programa de Ingeniería Informática y Tecnología en Sistemas de la Universidad de Caldas.

La investigación nace a partir de los resultados del proceso de investigación doctoral de uno de los autores, cuya pregunta problema es: ¿cómo diseñar e implementar estrategias metodológicas didácticas activas que permitan generar un proceso de inclusión tecnológica educativa que articule el B-Learning (Blended Learning) y el modelo Presencia, Alcance, Capacitación, Interacción y E-Learning (PACIE) en programas tecnológicos universitarios de Colombia, a través de las Tecnologías de la Información y la Comunicación (TIC)? 


\section{Investigación aplicada y desarrollo tecnológico en el contexto de los programas especiales de la Universidad de Caldas}

Los procesos de investigación aplicada, desarrollo tecnológico e innovación que se están estimulando en los centros de formación superior de la ciudad de Manizales hacen factible la implementación del proyecto, para lo cual se tienen a disposición los recursos físicos y tecnológicos del Sena y de la Facultad de Ingeniería de la Universidad de Caldas, que son dos organizaciones públicas que al estar articuladas al Sistema Universitario de Manizales (SUMA), tienen acceso y relación con las demás universidades de la ciudad.

La investigación, el desarrollo tecnológico y la innovación contribuyen a la mejora continua y al progreso de todos los sectores económicos de un país (Salas-Arbeláez, Solarte y Vargas, 2017). El sector empresarial, en especial cuando está organizado en cadenas productivas, redes, clústeres u otro tipo de esquemas de cooperación, es un ambiente propicio para la innovación, por su capacidad de interrelacionar factores como conocimientos científicos y tecnológicos en favor de clientes, usuarios y mercados (Riaño-Lozano, 2015).

La electrónica y las comunicaciones constituyen, sin lugar a duda, el área de mayor crecimiento y desarrollo en el entorno internacional. El desarrollo y la innovación de equipos para propósitos tanto industriales como domésticos son cada vez más altos.
Así pues, para cualquier persona que interactúe en nuestro medio, resulta claro que la electrónica constituye un factor fundamental para el desarrollo competitivo de un país.

Cabe anotar que, dentro del sistema productivo, las áreas de la electrónica como la automatización y el control de procesos constituyen el soporte de toda la dinámica productiva global, así como del administrativo y de gestión — sistemas de cómputo y de comunicación, entre otros-. En este sentido, se puede observar con claridad que las actuales tendencias evolutivas del contexto global han vinculado y posibilitado la aparición de nuevas empresas, cuya infraestructura es de base tecnológica y cuyas estrategias de gestión, de interconectividad e interoperabilidad ya no pertenecen a los microcosmos local y regional, ni siquiera al entorno nacional, sino que se perfilan como organizaciones e instituciones globales, como consecuencia de la dinámica de este contexto de cambio y crecimiento.

A partir de la experiencia docente a través de diferentes entidades de educación superior de la ciudad de Manizales, se han podido identificar los siguientes problemas:

- Desconocimiento por parte de los estudiantes de cuáles serían los contenidos temáticos que deben abordar para la investigación aplicada, desarrollo tecnológico e innovación.

- Limitación en los recursos tecnológicos para el desarrollo de las asignaturas de la investigación aplicada, desarrollo tecnológico e innovación.

- Escasa utilización de ambientes virtuales de aprendizaje por parte de los docentes y estudiantes. 
- Inexistencia de plataformas alternativas para el desarrollo de ambientes virtuales de aprendizaje.

- Inexistencia de una estandarización de contenidos del área de la investigación aplicada, desarrollo tecnológico e innovación.

- Deficiencia en la aplicación de técnicas didácticas activas en el desarrollo docente.

- Formación basada en libros tradicionales de metodología de la investigación, sin contextualizar el perfil profesional y ocupacional del programa tecnológico de la universidad

- Desarticulación entre el componente técnico y metodológico en la enseñanza de la investigación aplicada, desarrollo tecnológico e innovación.

A pesar de tener a nuestro alcance un bagaje en las herramientas tecnológicas y $\mathrm{TIC}$, los estudiantes de las áreas de especialidad de los programas tecnológicos de la Universidad de Caldas, desde el inicio hace más de quince años, nunca han participado en la iniciativa de creación de semilleros de investigación, pues no se sienten incluidos dadas sus dificultades en tiempo, espacio y recursos.

Considerando que la calidad educativa que se brinda en el nivel universitario debe ser igual para todos aquellos que estén interesados en obtener un título profesional, sin distinción de la modalidad de estudio que ellos elijan sea presencial, a distancia o virtual-, en esta investigación se propuso como objetivo general articular las TIC desde la virtualidad en los programas tecnológicos a distancia de la Universidad de Caldas —República de Colombia-, al incorporar en ellos fundamentos teóricos y prácticos que les permitan a los estudiantes desarrollar productos electrónicos aplicables y que fomenten la conformación e inscripción en los semilleros de investigación en dichos programas.

\section{Como objetivos específicos se definieron:}

a) Analizar los programas tecnológicos de la Universidad de Caldas, la selección de los grupos de estudiantes de los municipios de Manizales, Riosucio y La Dorada, los horarios de las actividades en campo y el espacio en el ambiente virtual de aprendizaje para realizar la intervención metodológica competencias en investigación aplicada e innovación.

b) Establecer los niveles de aprendizaje de las competencias en investigación aplicada e innovación de forma general para recolectar la información mediante la administración de una preprueba en cuatro secciones y la aplicación del programa solo al grupo experimental, mediante las 32 sesiones de aprendizaje en las que se incluyó el uso de las herramientas tecnológicas.

c) Diseñar técnicas didácticas activas que estimulen el pensamiento para la resolución de problemas simulados y reales utilizando el $B$-Learning y modelo PACIE, con el fin de lograr aprendizaje en las competencias de investigación aplicada e innovación, a través del ambiente virtual de aprendizaje que facilite la Universidad de Caldas. 
d) Analizar la información recolectada al concluir la aplicación del programa a las cuatro secciones para conocer si este mejoró el logro de aprendizaje en las competencias de investigación aplicada e innovación para los estudiantes.

e) Validar las estrategias metodológicas didácticas activas del proceso de formación aplicado utilizando $B$-Learning, el modelo PACIE y los recursos web 2.0 que se articularon.

Teniendo en cuenta lo anterior, se ha podido constatar que los estudiantes de cuarto, quinto y sexto semestre de la Tecnología en Electrónica de la Universidad de Caldas reciben una formación limitada y superficial en el tema específico de la investigación aplicada, desarrollo tecnológico e innovación. Como problema principal identificado se establece la estrategia de utilizar recursos digitales educativos a través del B-Learning, desarrollando un ambiente virtual de aprendizaje en la plataforma Moodle de la Universidad de Caldas para el fortalecimiento de las competencias en la investigación aplicada, desarrollo tecnológico e innovación de los estudiantes, actualizando los contenidos y estableciendo técnicas didácticas activas y objetos virtuales de aprendizaje pertinentes que se complementan, desde el componente técnico, con la creación una materia electiva en este tema para los programas tecnológicos en especial de electrónica.

Debidoalaausenciadealternativasquetienen losestudiantes para vincularse y participar en procesos de investigación formativa en contexto para la comunidad académica, y al no sentirse incluidos por sus dificultades en tiempo, espacio y disciplina, se hace evidente que los estudiantes requieren de herramientas para enfrentarse al entorno laboral por no saber formular ni ejecutar proyectos de emprendimiento e innovación. Por eso desde el mes de febrero de 2016 el ingeniero Fabio López, director de la Tecnología en Sistemas Informáticos, planteó la iniciativa de establecer una estrategia para la conformación y dinamización de los semilleros de investigación en los programas tecnológicos de la Universidad de Caldas. Para esto contarían con el acompañamiento y la asistencia técnica del Centro Metalmecánico Sena Distrito Capital representado un instructor y catedrático tutor de la Universidad de Caldas desde 2006, y líder del semillero de investigación E- InnovaCMM.

Durante los meses siguientes se estableció un plan de trabajo y en 2017 iniciaron jornadas de sensibilización y motivación con los docentes y estudiantes de las tecnologías de la Facultad de Ingeniería de la Universidad de Caldas, lo que generó participación y producción desde proyectos de aula de las asignaturas, y de los docentes en congresos nacionales e internacionales, producción de artículos científicos y la formulación y ejecución de seis proyectos de semillero. Así nació el semillero de investigación para la Tecnología en Sistemas Informáticos (TECSIS), con la participación de voluntarios. También se propuso crear e incorporar en el pensum de un programa de cuatro asignaturas donde se realice investigación formativa en contexto: Fundamentos de Investigación e Innovación 1, 2 y 3, y Fundamentos de Computación Embebida. 
Una vez estructurado el modelo de trabajo, se procedió a realizar la transferencia e involucramiento de los otros programas tecnológicos de la Universidad. En el mes de diciembre del mismo año, la directora de la Tecnología en Electrónica, Alba Lucía Cárdenas, aceptó involucrar a su programa y estructurar un plan de trabajo para 2018, permitiendo la creación del semillero de investigación RELEC para la Tecnología en Electrónica.

Durante 2018 se crearon las electivas Investigación Aplicada, Desarrollo Tecnológico e Innovación 1 y 2, en donde se logró formular 35 proyectos y realizar dos encuentros «La Institución Investiga - Investigación, Desarrollo Tecnológico e Innovación» en los municipios de La Dorada - 1. ${ }^{\circ}$ de mayo de 2018-y Manizales -1. ${ }^{\circ}$ de junio de 2018-. Para 2019 se hizo una transferencia tecnológica que permitió crear tres semilleros de investigación nuevos en la Universidad de Caldas para los programas especiales: GIRSPI, para la Tecnología en Higiene y Seguridad en el trabajo; SIMPROA, para la Tecnología en Agroindustria, y GITICLOG, para la Tecnología en Logística.

\section{Tecnologías de la información para la investigación}

Para referirse a las $\mathrm{TIC}$, se deben considerar las diversas acepciones que estas tienen en tanto que han ido mutando con el paso de los años; allí se ha incluido una variedad de elementos que han hecho que ingrese de una manera casi obligatoria en todos los ámbitos, desde lo académico hasta lo empresarial. La definición de TIC engloba diversos aspectos, que van desde ser herramientas teórico-conceptuales, soportes y canales que procesan, almacenan, sintetizan, recuperan y presentan información de la forma más variada, hasta la gestión de la información y el internet (Aprende en Línea, 2015).

Además, ser herramientas tecnológicas digitales que facilitan la comunicación e información por su accesibilidad e interconexión a la información online, incluyen una diversidad de características como interactividad, instantaneidad, innovación, interconexión, digitalización e inmaterialidad, multimedia, hipermedia, y conectividad (Grande, Cañón y Cantón, 2016; Rivera, 2011).

Desde otra vertiente, Ruiz, Mendoza y Ferrer (2014) refieren que las TIC son herramientas de gestión del conocimiento al mejorar el aprendizaje y hacerlo significativo para el estudiante, en cuanto facilitan el intercambio de información científica, pues permiten acceder a diversos contenidos lingüísticos y culturales de manera sincrónica y asincrónica entre estudiante y docente, con una abundante fuente de información.

Por esto, según Farroñay y Ancaya (2016, p. 35), estas herramientas permiten que el aula tradicional se convierta en un nuevo espacio, con actividades innovadoras, pues tienen el potencial de transformar los procesos de enseñanza y aprendizaje, como recursos y medios didácticos, al facilitar el acceso a bibliotecas virtuales, libros y artículos electrónicos, bases de datos, comunicaciones por correo electrónico, uso de redes sociales y blogs, entre otros (Izquierdo, Juárez y Salas, 2017). 
La comunicación virtual ha desplazado a la tradicional, gracias al desarrollo de las herramientas de comunicación virtual aplicables en diversos contextos y, sobre todo, en su uso como estrategia de aprendizaje en el aula. Los cambios originados por la irrupción de la tecnología en la educación desde el nivel básico hasta el superior han influenciado a las instituciones educativas para que integren las tecnologías al proceso de aprendizaje con el fin de mejorarlo (Chávez, Del Toro y López, 2017).

Dentro de estas tecnologías, según Chávez, Del Toro y López (2017): «El blog constituye una herramienta de comunicación virtual usada con frecuencia en la educación» (p. 48). Según Gálvez (2010, citado en Larreal, 2015):

\section{Es una página web en la que se puede integrar texto, imágenes, hipervínculos, vídeos y muchos otros objetos, permitiendo que los usuarios puedan hacer labor de autores o escritores, puesto que mediante esta herramienta se puede contener mensajes de una o varias personas respecto a un tema específico y quienes lean el blog pueden participar mediante sus aportaciones o cuestionamientos, promoviendo la reflexión individual y colectiva. (p. 723)}

Los recursos educativos digitales como los blogs, articulados con las estrategias de enseñanza-aprendizaje de los semilleros de investigación en las diversas plataformas de administración de aprendizaje —como NeoLMS y Moodle-, constituyen una herramienta valiosa para complementar los diversos cursos que se orientan desde el campus virtual de la Universidad de Caldas.
Las redes sociales son otro tipo de mediaciones desde la virtualidad. Al respecto, Porras (2017) destaca el uso de redes sociales o comunidades como potenciadores de los procesos de enseñanza-aprendizaje al emplear recursos web 2.0 para establecer contactos, compartir información, jugar, ejecutar actividades de colaboración, chatear, compartir aficiones y comprar y vender productos. WhatsApp y Blogger conforman un ciberespacio adecuado para comunicarse y una estrategia que gestiona los procesos educativos del siglo XXI, al facilitar y permitir el aprendizaje colaborativo y el intercambio de información en comunidades de aprendizaje y cooperación.

Para Izquierdo, Juárez y Salas (2017), es necesario reflexionar sobre la forma en que la docente incentiva a sus estudiantes para que empleen las TIC en todo su proceso investigativo y cómo se vale de ellas para generar una cultura digital con la apropiación de estos medios como software y las bases de datos especializadas en cada uno de los elementos del proceso de investigación, con el fin de contribuir y enriquecer las acciones de colaboración entre grupos. Estos medios brindan a cada docente la experiencia de integrar interactividad en sus prácticas educativas, desarrollar repositorios de trabajo para los estudiantes, compartir recursos didácticos para utilizarlos en clase y motivar e incentivar su ejercicio educativo (Rojas, 2017).

Según Morales-Salas y Pereida-Alfaro (2017), el aprendizaje significativo requiere de estrategias didácticas cuyo centro es el aprendizaje del estudiante. Estas son herramientas pedagógicas que contribuyen a un aprendizaje profundo y permanente, propician el desarrollo del pensamiento crítico y establecen habilidades cognitivas y actitudes desde una perspectiva constructivista y significativa. 
En cuanto a la educación apoyada en el B-Learning, TurpoGebera (2013) muestra que la convergencia pedagógica y tecnológica surge como una presencia educativa en la que confluyen la presencialidad y virtualidad como un todo. Un ecosistema educativo que diluye las fronteras artificialmente instauradas e instituye un flujo de continuidad entre los escenarios y componentes que la configuran. Este salto evolutivo es propio del proceso de aprehensión progresiva que los agentes y sujetos pedagógicos van configurando conforme se comprende su importancia y transcendencia en los procesos formativos. Esta es una dinámica presente y que amerita ser reconocida. Un proceso que deviene de las sucesivas transiciones por las que discurre el Blended Learning.

La aplicación de las TIC en los procesos educativos, el diseño e implementación de aulas virtuales bajo diversas plataformas, como Moodle, Blackboard y Neolms, entre otros, y la facilidad de diversos procesos tecnológicos han llevado a reproducir lo real en lo virtual sin usar metodología alguna. De ahí surge una nueva metodología de trabajo en línea a través de un campus virtual llamada PACIE, creada por el Ing. Pedro Camacho (2009) —fundador de la Fundación para la Actualización Tecnológica de Latinoamérica (FATLA)—, que permite manejar mejor ese proceso de transición, tanto en los espacios presenciales cotidianos como en los institucionales; guiar por internet el proceso de enseñanza-aprendizaje, con la implementación de recursos didácticos usados en clase.

Un ambiente virtual de aprendizaje es un espacio de acceso a información por parte de alumnos que interactúan entre sí y reciben la guía y orientación del docente en su proceso de aprendizaje. Reciben ese nombre al no requerir presencia física ni sincronía en un tiempo determinado. Domínguez, Rama, y Rodríguez (2013) afirman que «[...] surge la modalidad virtual con un nivel de incorporación de las TIC amplio, llegando a la implementación de entornos virtuales de aprendizaje (EVA), entendidos estos como ambientes de aprendizaje mediados por tecnologías» (p. 263).

Es posible acceder a los ambientes virtuales de aprendizaje los 365 días del año, las 24 horas del día, sin límite de tiempo ni espacio. Allí los estudiantes tienen disponibles herramientas como foros, chats y correo electrónico, entre otros, al igual que la posibilidad de acceder cuantas veces deseen a los contenidos del curso como información, videos y audios, entre otros. Así, «un Ambiente Virtual de Aprendizaje (AVA) [se puede definir] como el conjunto de entornos de interacción sincrónica y asincrónica donde, con base en un programa curricular, se lleva a cabo el proceso Enseñanza-Aprendizaje» (Calle-Valencia y López-Becerra, p. 7).

Calle-Valencia y López-Becerra por su parte, sostienen que los objetos virtuales de aprendizaje (OVAS) son:

Contenido del curso que ha sido adaptado de una versión física a virtual, ya sea en video, texto, gráfico o audio, de tal manera que se mejore la experiencia del aprendizaje, haciendo de estas unidades digitales de información de tipo interactivo utilizados en el contexto pedagógico. (p. 6)

Según Chacón-Castro, Moreno-Barragán y GómezGamboa (2014): 
Todo acto educativo implica acciones comunicativas entre docente y estudiantes, quienes comparten información y la procesan para generar conocimiento. En el ambiente de formación (aula), actividades como la exposición y discusión oral, la lectura de textos impresos, la ejercitación y la práctica en laboratorio se apoyan con materiales educativos como tablero, libros, documentos y manuales impresos. Estos materiales sirven como mediadores en el proceso enseñanzaaprendizaje, para comunicar los contenidos y facilitar su comprensión y apropiación.

Con las Tecnologías de la Información y la Comunicación (TIC) se producen medios integrando texto, imagen, audio, animación, video, voz grabada y elementos de software, para almacenarlos en computadores - llevarlos a Internet para ser leídos desde un computador o un dispositivo móvil. A estos medios se les conoce como medios digitales por el hecho de estar representados en un lenguaje binario, compuesto por dígitos (0 y 1), propio de los computadores. (Zapata, 2012, citado por Chacón-Castro, Moreno-Barragán, y Gómez-Gamboa, 2014)

García (2010) sostiene que un recurso educativo digital (RED) se define como cualquier tipo de contenido que tiene una clara intencionalidad educativa. Ese contenido debe ser digital y contener metadatos que permiten su descripción.

Para Maldonado, Siguencia y Carvallo (2015), los metadatos son el conjunto de información provista con el fin de describir un material educativo, y constituyen una de las características más relevantes de los RED, los cuales permiten identificar cada recurso; se definen como datos sobre los datos, al describir aquellas características que detallan más profundamente cada uno de estos recursos digitales.
López-Guzmán y García-Peñalvo (2006) afirman que los RED se alojan con sus metadatos en repositorios - bibliotecas digitales especializadas-, orientados a facilitar la búsqueda y recuperación de los recursos, de forma que puedan ser utilizados en diferentes ambientes de E-Learning. En este sentido, algunas propuestas para evaluar material educativo desarrolladas por Tabares-Morales (2014) se enfocan en analizar características de los RED desde diferentes perspectivas, tales como la de expertos y usuarios que interactúan con los recursos. Así mismo, es posible realizar procesos de evaluación automáticos orientados a la gestión del RED y sus metadatos. Se pretende evaluar el grado de cumplimiento que tienen los objetos respecto a un conjunto de características y criterios que pueden llegar a definir su nivel de calidad. Los resultados que entrega el modelo propuesto sirven de insumo para la organización de resultados de búsquedas, retirar recursos de repositorios y definir planes de mejoramiento para aquellos materiales educativos que no cumplan con elementos mínimos desde el punto de vista educativo, de contenido, funcional y de metadatos (González-Ruíz, 2016).

Las técnicas didácticas activas -e incluso métodos y estrategias didácticas-buscan aplicar el principio de actividad en la formación, de tal modo que los aprendices se conviertan en protagonistas de la actividad y de su propio aprendizaje. Son representativas de la extensa relación de técnicas que responden a esta caracterización las siguientes técnicas, que se presentan siguiendo un criterio de complejidad creciente: 
Estudio de casos: presenta a los aprendices un episodio - situación concreta con amplia información sobre las circunstancias que lo envuelven con el fin de analizarlo, resolverlo, elaborar conclusiones y proponer posibles vías de acción para su resolución.

Juegos de simulación: Son «reproducciones simplificadas de la vida real en las que se elimina la mayor parte de la información irrelevante, se secuencian las fases y se permite a los aprendices ser los verdaderos actores de la situación, enfrentándose a la necesidad de tomar decisiones y valorar sus resultados» (Martín, 1983, citado en Oña-Quisatasig, 2012).

Análisis de objetos, sistemas, servicios: puede ser utilizado en cualquier contexto en el que se requiera dicho análisis, aunque tiene una mayor aplicación en ámbitos productivos/industriales. Por su parte, el análisis de servicios/ actividades comerciales - sin ser excluyente- cobra un mayor significado en el desarrollo de procesos formativos del ámbito de comercio y servicios

Las universidades, como fuentes de saber, requieren incorporar en sus planes de gestión la transferencia de conocimiento para acercarse de forma comprensiva a los clientes naturales, consumidores de información y de conocimiento. Sin embargo, es paradójico que las universidades consideren que en los programas tecnológicos no se hace investigación, motivo por el cual no se estimulan la creación y permanencia de semilleros de investigación, sin dimensionar la capacidad de llevar a cabo desarrollos tecnológicos y producción científica de estos programas, contribuyendo al desarrollo integral de los estudiantes y comunidad.

El estudio «Tecnología para la transformación y el mejoramiento de la educación. Experiencias de éxito y expectativas de futuro", presentado en octubre de 2014, reafirma las conclusiones de un estudio anterior de la Unesco, el cual pone de presente que el pilar fundamental de la calidad educativa son las competencias docentes, siendo las TIC una de ellas, y en las cuales las digitales facilitan el desarrollo de las otras.

\section{Metodología}

La metodología empleada corresponde a una investigación cualitativa analítica, de carácter descriptivo y corte transversal, a partir de la cual se pretende cimentar proyectos posteriores que permitan profundizar en el conocimiento científico de unos campos específicos de conocimiento, aplicando el B-Learning a través del modelo PACIE, mediante los semilleros de investigación E-InnovaCMM del Centro Metalmecánico Sena Distrito Capital. Los materiales y herramientas empleados son recursos educativos digitales como edublosgs, wikis, videos, guías didácticas, mapa de empatía, escenarios de uso y RAE, entre otros.

Se ha logrado divulgar productos como capítulos de libros, artículos científicos y recursos educativos digitales multimedia, virtuales y de audio, los cuales han servido de modelo para 
constituir transferencias tecnológicas para la estructuración de proyectos de investigación aplicada e innovación, usando la metodología de marco lógico, estructuración y divulgación a través de posters y artículos científicos desde los semilleros de los grupos de Investigación ReNuevaTe Ciencia, Tecnología e Innovación, de la Universidad de Caldas; GICEMET, del Centro Metalmecánico Sena y BIEMARC, de la Universidad de La Guajira. También, para consolidar la producción científica en otros centros de formación de la Regional Distrito Capital y Quindío.

\section{Conclusiones}

El desarrollo de este proyecto ha contribuido a la inclusión educativa de los jóvenes desde las provincias profundas de los departamentos de Caldas, Cundinamarca y La Guajira, en donde hacen presencia las instituciones vinculadas. Para este caso, se pudo aplicar la transferencia tecnológica de las estrategias didácticas activas y recursos educativos digitales desarrollados desde el semillero de investigación E-InnovaCMM del Centro Metalmecánico Sena Distrito Capital, con el acompañamiento y la colaboración del grupo de investigación BIEMARC, de la Universidad de La Guajira, los cuales iniciaron con el semillero de la Tecnología en Sistemas Informáticos (TECSIS), conformado en febrero de 2017; luego, el semillero de investigación RELEC, conformado en febrero de 2018 y para 2019 otros tres semilleros de los programas tecnológicos de la Facultad de Ingeniería de la Universidad de Caldas.
El desarrollo de recursos educativos digitales para el ecosistema tecnológico del Centro Metalmecánico ha facilitado el aprendizaje significativo y el uso en todos los niveles de formación -tecnológico, técnico y complementario-.Para 2019, en el Centro Metalmecánico, mediante el instructor Luis Devia, se realizó la aplicación de esta investigación, a través de formación complementaria presencial, combinando el B-Learning y los recursos de la plataforma NeoLMS del semillero de investigación para 240 aprendices del Instituto Técnico Industrial Francisco José de Caldas, desde el semillero de investigación e innovación E-InnovaCMM como eje articulador en la virtualidad.

Se logró acompañar y asesorar la creación de los semilleros de investigación para los programas tecnológicos de la Universidad de Caldas RELEC - Tecnología en Electrónicay TECSIS - Tecnología en Sistemas Informáticos-.Se conformó la mesa de trabajo Mesa 5_01, La E-inclusión y las Tecnologías Apropiadas para la Interacción Social en Infancias y Juventudes, donde se participará en calidad de organizadores durante la III Bienal Internacional de Infancias y Juventudes, en el cual se presentaron y evaluaron diecisiete ponencias en el tercer trimestre de 2018.

El avance de este proyecto, en uno de los casos desarrollados para el ecosistema tecnológico desde la virtualidad en la enseñanza de la automatización, permitió crear recursos educativos digitales (RED) que han facilitado el aprendizaje significativo de los aprendices adscritos a los cursos virtuales, como Bombas Rotativas Mecánicas, Bombas Lineales Mecánicas, Diseño de Productos Electrónicos con Microcontroladores, PLC I —Controladores 
Lógicos Programables 1-, Aplicaciones de los PLC en la Automatización de Procesos industriales y Electrónica Digital Secuencial. El nivel de complejidad técnica de estos cursos requiere de estrategias didácticas activas mediadas por las TIC han permitido cambiar los bajos niveles de certificación y altos índices de deserción que en ellos se presentaban, alcanzando niveles de certificación de entre el $25 \%$ y el $40 \%$, frente a los que se tenían sin aplicarlos, que eran del $10 \%$. Uno de los RED realizados es el EdublogPLC, podcast motivacionales y de apoyo al tema, alojados en SoundCloud, y videos tutoriales en los canales de Vimeo y YouTube.

\section{Referencias}

Aprende en Línea. (8 abril de 2018). Las TIC como apoyo a la educación. Recuperado de http://aprendeenlinea.udea.edu. $\mathrm{co} / \mathrm{ms} /$ investigacion $/ \mathrm{mod} /$ page/view.php?id=3118

Calle-Valencia, D. C.; López-Becerra, M. H. (2018). Paces Imperfectas para el perdón y la reconciliación en Colombia. Corima. Revista de Investigación en Gestión Cultural, 5(3), 1-16. DOI: https://doi.org/10.32870/cor.a3n5.7202

Chacón-Castro, S.; Moreno-Barragán, A.; Gómez-Gamboa, W. A. (2014). Recurso educativo digital para estudiantes de preescolar como apoyo en los procesos lecto-escriturales (Tesis de doctorado). Corporación Universitaria Minuto de Dios. Bogotá, Colombia. Recuperado de http://hdl.handle. net/10656/3183

Chávez, J.; Del Toro, M.; López, O. (2017) Blog, correo electrónico y foros temáticos: su uso, dominio y actitud en estudiantes de educación medio superior de México. Revista Hamut'ay, 4(2), 45-54. DOI: https://doi.org/10.21503/hamu.v4i2.1471
Domínguez, J.; Rama, C.: Rodríguez, J. (2013). La educación a distancia en el Perú. Chimbote. Perú: Universidad Católica los Ángeles de Chimbote. Recuperado de https://bit.ly/38UiahN

Farroñay, P.; Ancaya, M. (2016) Gestión administrativa y conocimiento de las TIC en docentes de educación primaria de las instituciones educativas Innova Schools de San Juan de Lurigancho y Ate. Revista Hamut'ay, 3(1), 31-45. DOI: https:// doi.org/10.21503/hamu.v3i1.998

García, E. (2010). Materiales Educativos Digitales. Blog Universia. Recuperado de http://formacion.universiablogs. net/2010/02/03/materiales-educativos-digitales/

González-Ruiz, L. M. (2016). Propuesta para evaluar la calidad de objetos de aprendizaje mediante el uso de ontologías (Tesis de doctorado). Universidad de Alicante, España. Recuperado de https://rua.ua.es/dspace/bitstream/10045/53886/1/tesis gonzalez ruiz.pdf

Grande, M.; Cañón, R.; Cantón, I. (2016). Tecnologías de la información y la comunicación: evolución del concepto y características. International Journal of Education Research and Innovation, 6, 218-230.

Izquierdo, B.; Juárez, G.; Salas, B. (2017). Importancia de las tecnologías en los trabajos de investigación: una experiencia en los estudiantes de Licenciatura, Área económicoadministrativa de la Universidad Veracruzana-México. Revista Hamut'ay, 4(1), 9-17. DOI: https://doi.org/10.21503/hamu. v4i1.1392

Larreal, B. (2015). Herramientas de comunicación para el desarrollo de la inteligencia lógica matemática. Revista Opción, (3), 715-734. 
Rubén Darío Cárdenas-Espinosa / Luis Alfonso Devia-Caicedo / Darwin Dubay Rodríguez-Pinto

Claudia María Martínez-Zuluaga / Fabio Andrés López-Salazar / Jhon Jairo Ruiz-Salazar

López-Guzmán, C.; García-Peñalvo, F. J. (2006). Repositorios de objetos de aprendizaje: bibliotecas para compartir y reutilizar recursos en los entornos e-learning. Biblioteca universitaria, 9(2).99-107.

Maldonado, J. J.; Siguencia, J.; Carvallo, J. P. (2015). Repositorios educativos: estudio de la situación actual y estrategia para mejorar su uso efectivo en las universidades ecuatorianas. Revista Iberoamericana de Tecnologías del/da Aprendizaje/ Aprendizagem, 3(2), 85-92.

Morales-Salas, R. E.; Pereida-Alfaro, M. A. (2017). Inclusión de estilos de aprendizaje como estrategia didáctica aplicada en un AVA. Campus Virtuales, 6(1), 67-75.

Oña-Quisatasig, J. R. (2012). Diseño de un proyecto práctico de tareas dirigidas a estudiantes de 6 a 12 años del barrio Zumbalica, parroquia Eloy Alfaro, cantón Latacunga durante el periodo 2009-2010 (Tesis de Licenciatura). Universidad Técnica de Cotopaxi, Ecuador. Recuperado de https://bit. ly/3nBO6vr

Porras, I. (2017). Redes sociales, Facebook y blog según los estilos de aprendizaje en cursos E-Learning. Revista Hamut'ay, 4(1), 60-74. DOI: https://doi.org/10.21503/hamu.v4i1.1395

Riaño-Lozano, C. M. (2015). Diseño de un Modelo de Transferencia del Conocimiento para el Manejo del Sector Autopartista en Bogotá (Tesis de maestría). Universidad Distrital Francisco José de Caldas, Bogotá. Recuperado de http://hdl.handle. net/11349/7238

Rivera, J. (2011). Impacto de las tecnologías de información y comunicación en los procesos de enseñanza-aprendizaje. Investigación Educativa, 15(27), 127-137.
Rojas, M. (2017) Los recursos tecnológicos como soporte para la enseñanza de las ciencias naturales. Revista Hamut'ay, 4(1), 85-95. DOI: https://doi.org/10.21503/hamu.v4i1.1403

Ruiz, N.; Mendoza, M.; Ferrer, L. (2014) Influencia de las tecnologías de la información y comunicación en los roles e interrelaciones entre estudiantes y docentes en programas presenciales de educación superior. Hallazgos, 11(22), 435-454. DOI: https:// doi.org/10.15332/s1794-3841.2014.0022.22

Salas-Arbeláez, L.; Solarte, M. G.; Vargas, G. M. (2017). Efecto de la cultura organizacional en el rendimiento de las PYMES de Cali. Suma de Negocios, 8(18), 88-95. DOI: https://doi. org/10.1016/j.sumneg.2017.11.006

Tabares-Morales, V. (2014). Modelo por Capas para Evaluación de la Calidad de Objetos de Aprendizaje en Repositorios de Objetos de Aprendizaje (Tesis de maestría). Universidad Nacional de Colombia, Medellín. Recuperado de https://bit. ly/3kOyjax

Turpo-Gebera, O. (2013). Perspectiva de la convergencia pedagógica y tecnológica en la modalidad blended learning. Universidad Peruana Cayetano Heredia. Revista de Educación a Distancia (RED), (39)1-14. 